\title{
The Control System Design of Hydraulic Hybrid Vehicle Based on CAN Bus
}

\author{
Shigang Li ${ }^{*}, 1$, Dandan Liu ${ }^{1}$ and Bin Xie ${ }^{2}$ \\ ${ }^{I}$ College of Mechanical and Electrical, Beijing Union University, Beijing, China \\ ${ }^{2}$ College of Engineering, Agricultural University China, Beijing, China
}

\begin{abstract}
The hybrid vehicle is a new type of green and energy efficient vehicle. Hydraulic accumulator and variable pump/motor make up the break energy regeneration utilization system, which is the core of hydraulic auxiliary driving unit. The hybrid drive system is composed of hydraulic auxiliary driving unit and the original vehicle drive system. As it relates to the recovery of the braking energy and the situation of two kinds of power-driven vehicle, it brings complexity to the hybrid distribution strategy, and an efficient energy control system is necessary. Based on CAN bus technology, using the CAN2.0B communication protocol for the system design, the core hardware system is composed of PC/104 computer and CAN communication card, the key nodes of hydraulic auxiliary drive unit use DSP chip TMS320LF2407. Experiments show that the system has a high communication reliability and high accuracy of dynamic coupling, the safety of vehicles and the best energy matching.
\end{abstract}

Keywords: CAN bus, Control system, DSP, Dynamic coupling, Hydraulic hybrid vehicle, A/D module.

\section{INTRODUCTION}

With the social development, green and energy efficient vehicle is an important direction of automobile industry. With the use of energy regeneration technology, hydraulic hybrid vehicle shows great advantage in vehicle emission reduction and energy saving. Energy regeneration technology has broad application prospects. With characteristics of being real-time, high error detection capability, simple connection, strong expansibility $[1,2]$ and so on, CAN bus technology is suitable for complex dynamic matching and network security requirement of hydraulic hybrid vehicle [3]. Therefore, in this text, the control system design is based on CAN technology.

\section{CONTROL SYSTEM COMPONENTS}

The control system of hydraulic hybrid vehicle is a distributed and embedded field bus control system, with the smaller PC104 industrial computer as the host computer; the system could achieve high level management and optimal control. The master-slave response communication of 250 kbps baud rate is used in vehicle bus layout, although this resulted in the decreased utilization of channel, but the realtime data transmission between host computer and critical nodes of power source is guaranteed. The control system structure of hydraulic hybrid vehicle based on CAN bus communication is shown in Fig. (1).

\footnotetext{
*Address correspondence to this author at the College of Mechanical and Electrical, Beijing Union University, North Fourth Ring Road No. 97, Chaoyang District of Beijing, 100021, China; Tel: +86 13810276385; E-mail: lishigang2523@163.com
}

\section{CONTROL SYSTEM NOD OF HYDRAULIC HYBRID VEHICLE BASED ON CAN BUS}

\subsection{Software and Hardware Design of Key Nodes in Variable Pump/Motor}

As shown in Fig. (1), the network nodes of hydraulic hybrid vehicle control system are mainly: PC, engine, hydraulic auxiliary drive unit, the accelerator pedal/break pedal and etc. PC: the system is composed of PC/104 computer + CAN communication card, with monitors, printers, serial communication, CAN bus communication interface. The PC nod runs on WINCE, which is an embedded operating system [4, 5]. Hydraulic auxiliary driving unit: this system is composed of digital signal processor (DSP), with collectors, communication, digital input/output interface; the TMS320LF2407A node controller (lower PC) is used to ensure the timely fault detection and rapid sophisticated smoothing algorithm. In addition, the CAN controller of TMS320LF2407A supports CAN2.0 protocol, it can time programming bit and configure interrupt programming, with bus wake-up function and automatic remote reply function [8]; the other nodes use the system composed of SCM 8051 and chip P89C54UFPN as slave computer, with the acquisition, communications, digital input/output interfaces [6,7].

The composition diagram of Hydraulic auxiliary drive unit is shown in Fig. (2), mainly consists of DSP chips, power circuit, ADC interface circuit, CAN bus interface circuit, PWM output drive circuit, I/O interface circuit and other components. Key nodes of hydraulic pump/motor use DSP chips (TMS320LF2407A of TI Company), with advantages of high speed and low power consumption, there 


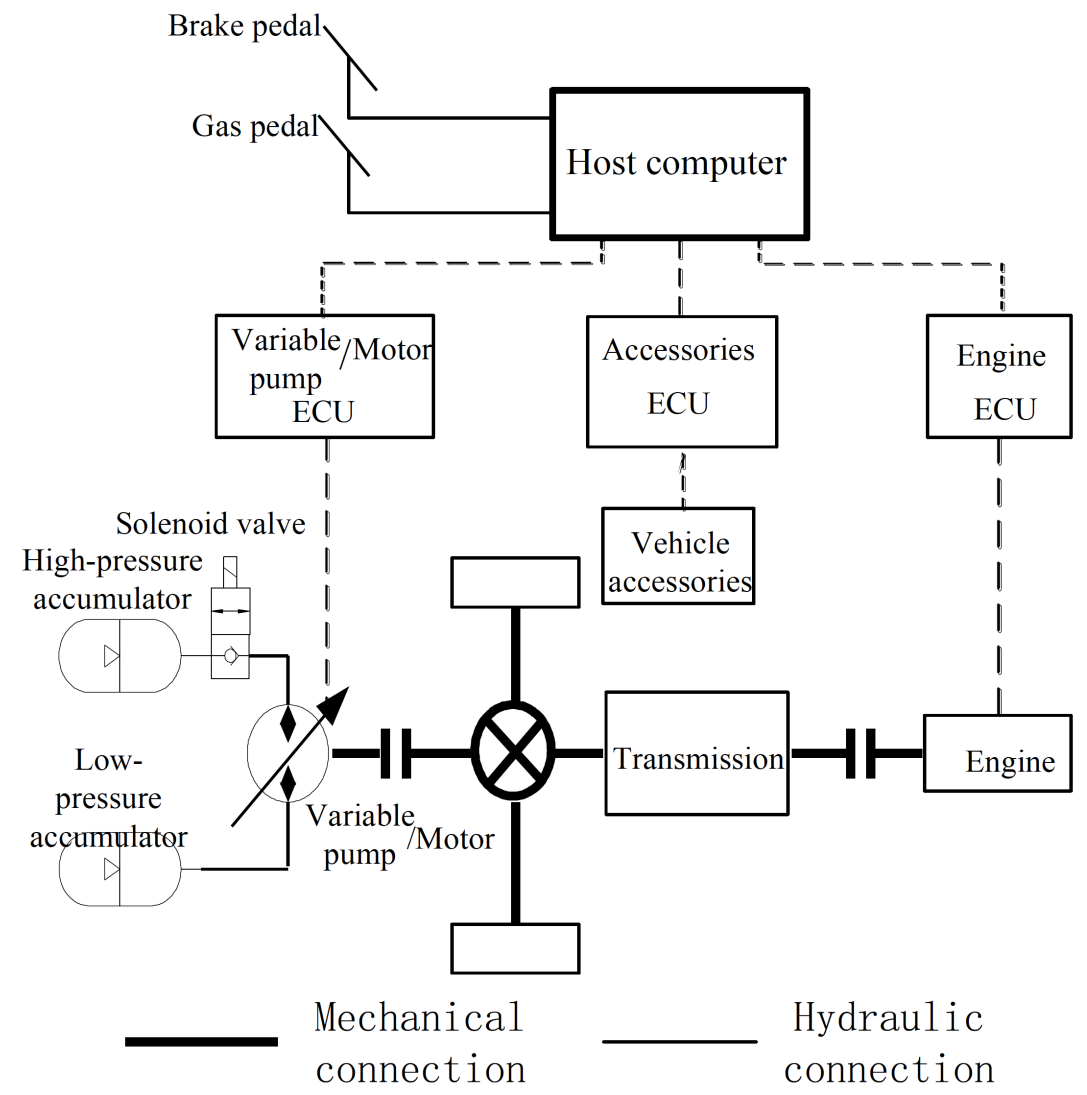

Fig. (1). The diagram of based on CAN Bus in vehicle control system.

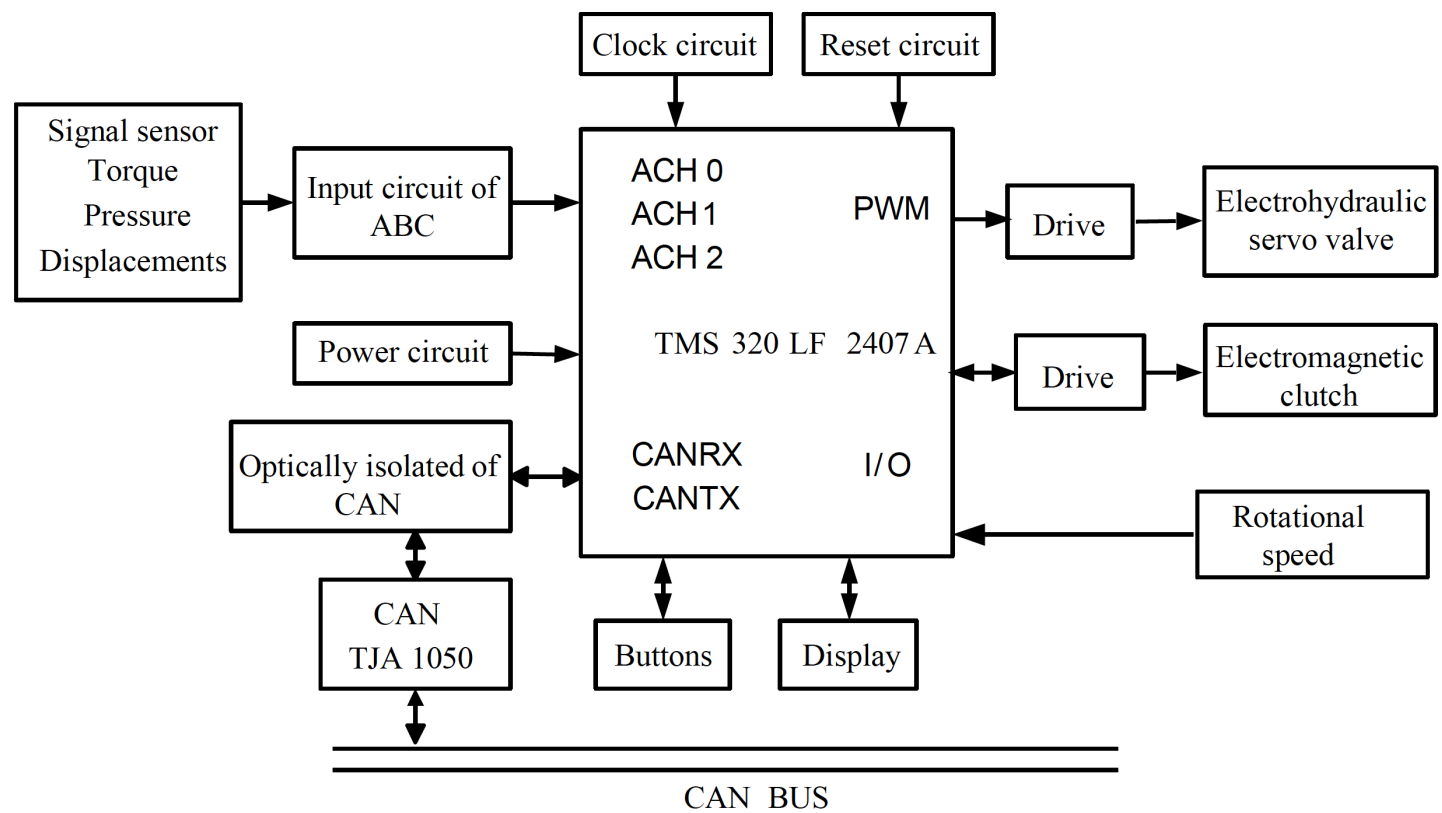

Fig. (2). The circuit diagram of node in Hydraulic pump/motor.

is a CAN controller in this chip. The CAN controller in TMS320LF2407A supports CAN2.0B protocols, it works at standard mode and extended mode, receives and sends data with E-mail, and configures the receiving mask words for receiving E-mail. This controller can do configuration of programmable interrupt and time the programming bit, with bus wake-up function and auto-reply function of remote request. When sending an error or losing arbitration, it does an automatic retransmission, and there is a bus error diagnostics, also can be in the self-test mode [8].

The overall software program of hydraulic pump/motor is divided into three levels: the main loop foreground daemon, the interrupt processing background daemon and function module background module. With this method, each functional module is relatively independent, which is very 


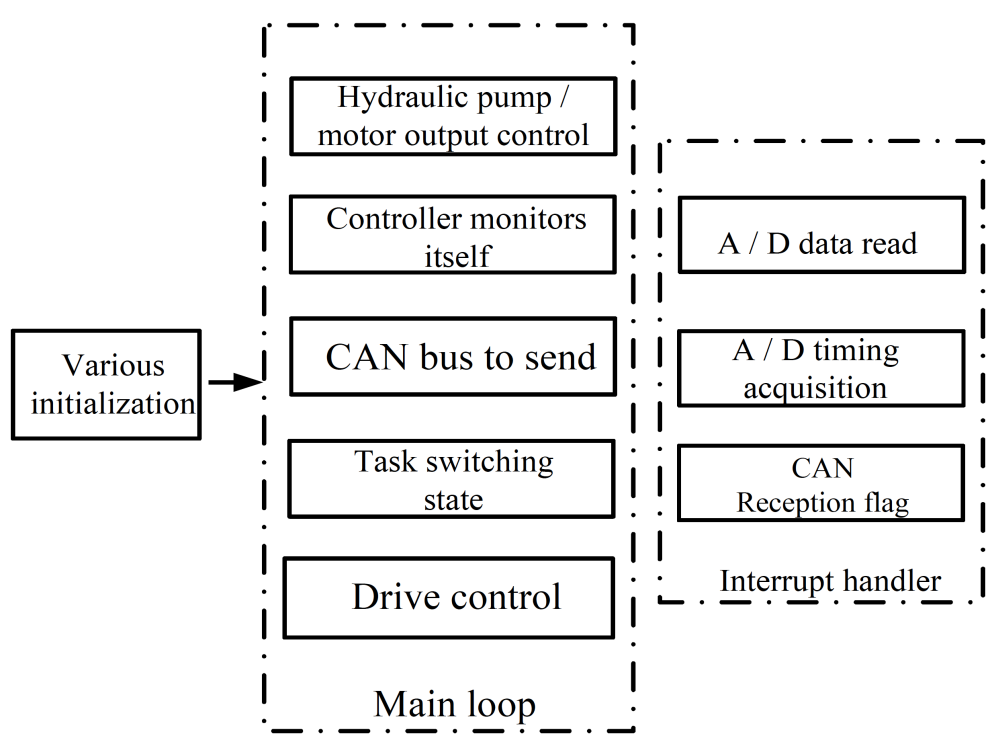

Fig. (3). The subsystem block diagram in main program of hydraulic auxiliary drive unit.

beneficial for debugging. For the embedded controller, the software part is of its core. Software design should first guarantee the stability of the system, and ensure the perfect function, system maintainability and module programming. This software is based on this design and works at foreground to background manner. The main loop of foreground system mainly includes software implementation of control strategy and software control of each sub-module. The background system is mainly responsible for the interrupt processing and control based on physical layer. There is an interface between the background and foreground system, which makes the software easy to modify. The main framework of the key nodes in hydraulic pump/motor is shown in Fig. (3).

\subsection{Software and Hardware Design of Accelerator/Break Pedal}

The controller of accelerator/brake pedal use P89C54UFPN chip compatible with 8051. P89C54UFPN is an 8 bits single chip produced by Philips Company, and its core microcontroller is $80 \mathrm{C} 51$ which uses the advanced
CMOS technology. CPU clock frequency is up to $33 \mathrm{MHz}$, with $16 \mathrm{~K}$ FLASH, and 256 bytes SARAM. The P89C54UFPN chip can address the program memory and data memory separately. The address space is $64 \mathrm{~K}$, double DPTR register, three 16-bits timers, four 8 bits I/O ports, enhanced full-duplex UART, and serial programming (ISP) (Fig. 4).

The software mainly consists of three parts: initialization subroutine, safe handling subroutine, and data receiving subroutine. The initialization process flow of $\mathrm{A} / \mathrm{D}$ module in P89C54UFPN is shown in Fig. (5).

\section{TEST RESULT AND ANALYSIS}

The communication test of control system is based on these conditions: CAN bus communication baud rate is set to $100 \mathrm{~K}$; communication distance is 20 meters, and data update cycle is $0.05 \mathrm{~s}$. There are at least four nodes at work, the multi-node communication is in lab, and the controller works continuously for 48 hours, without error.

In the bench test, using hydraulic auxiliary drive control strategy, the maximum pressure of hydraulic accumulator is

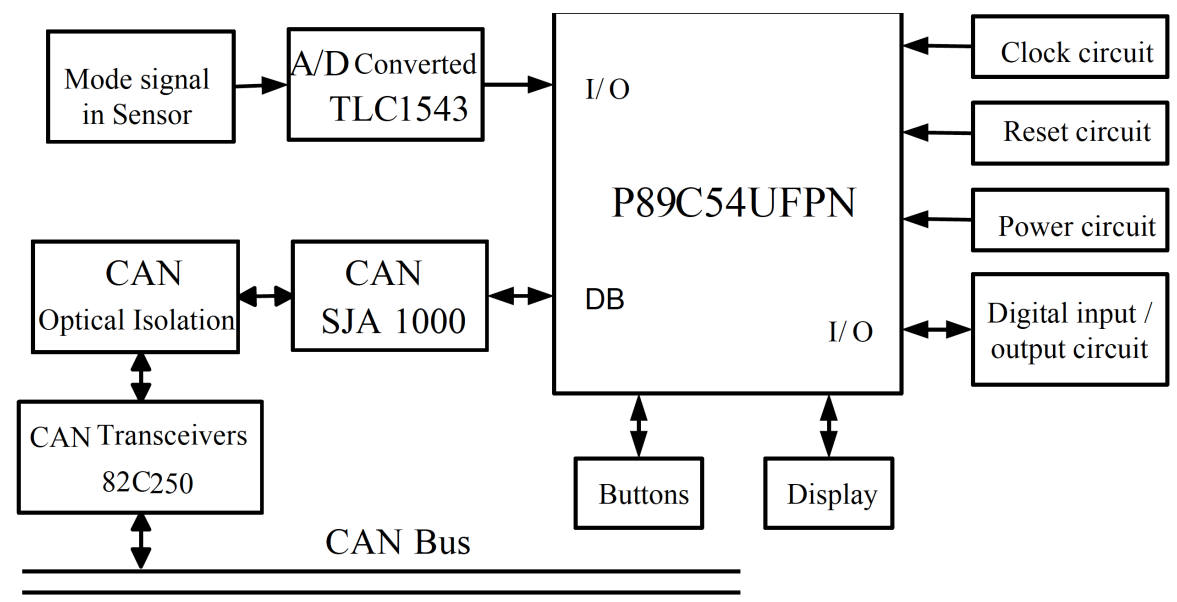

Fig. (4). The schematics configuration of nodes in P89C54UFPN. 


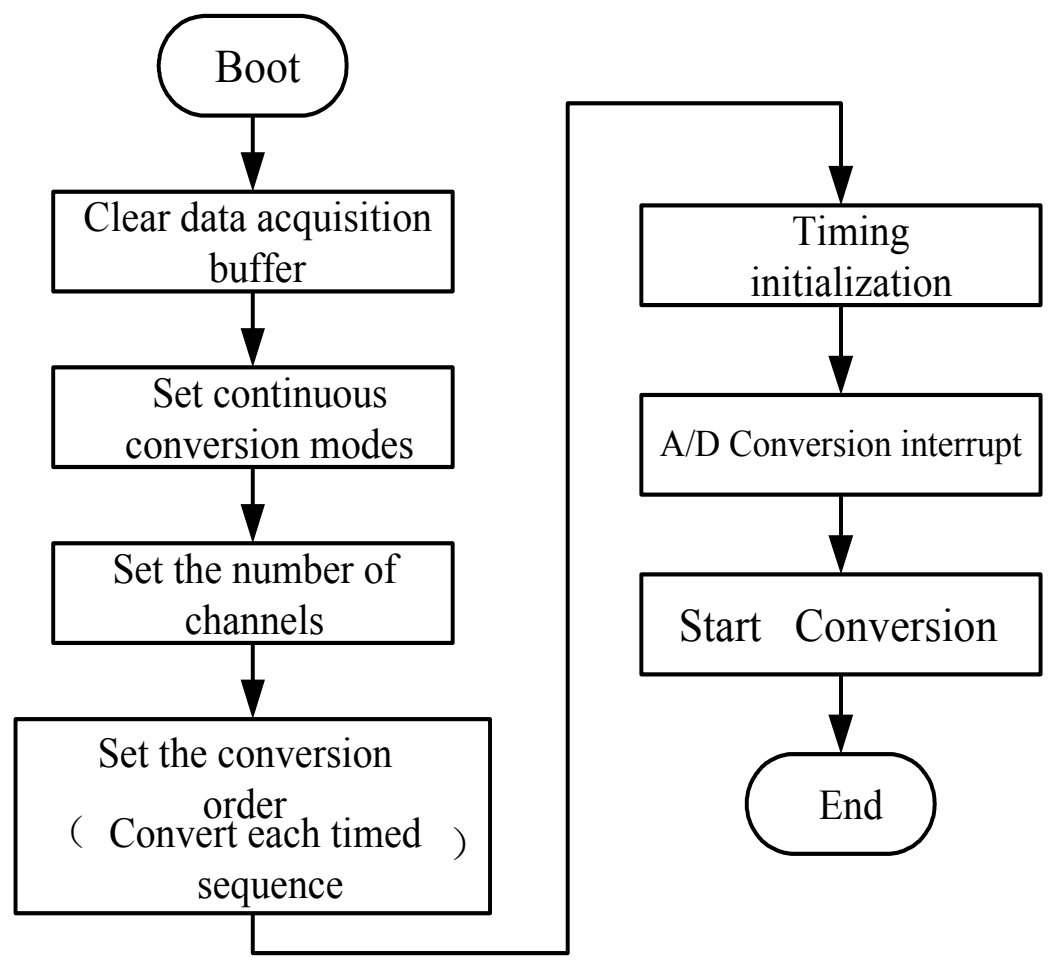

Fig. (5). The A/D module initialization procedure flow chart in P89C54UFPN.

31.5 Mpa. Before the test, load 125 N.m of torque with eddy current dynamometer, and observe the speed sensor of engine. Adjust the throttle opening degree to stabilize the engine speed at about $1500 \mathrm{r} / \mathrm{min}$ (fix throttle pedal to make sure the position does not change). Increase the output torque of eddy current dynamometer to 250 N.m, gradually. At this point, join the electromagnetic clutch; open the electromagnetic valve switch of hydraulic accumulator, so that the variable pump/motor works as motor and outputs power. Speed curve of the engine is shown in Fig. (6), the curve shows that, when the output torque of eddy current dynamometer increases from 125 N.m to 250 N.m, the speed of engine decrease from $1500 \mathrm{r} / \mathrm{min}$ to $950 \mathrm{r} / \mathrm{min}$ in a short time, respectively. At this point, join the electromagnetic clutch, and variable pump/motor exports power. The curve shows that, the speed of engine begins to increase, and becomes steady when it reaches $1500 \mathrm{r} / \mathrm{min}$. As shown in the curve, variable pump/motor is involved in work (about 4 second), the engine speed does not decrease suddenly, and it shows that, hydraulic pump/motor does not become a system load. In contrast with its continuous output power, the engine speed continues to increase rapidly until the system load is 125 N.m. Therefore, we can conclude that the design of the control system is successful. With the system, we can control the coupling of two power source precisely, and balance the engine's power efficiently.

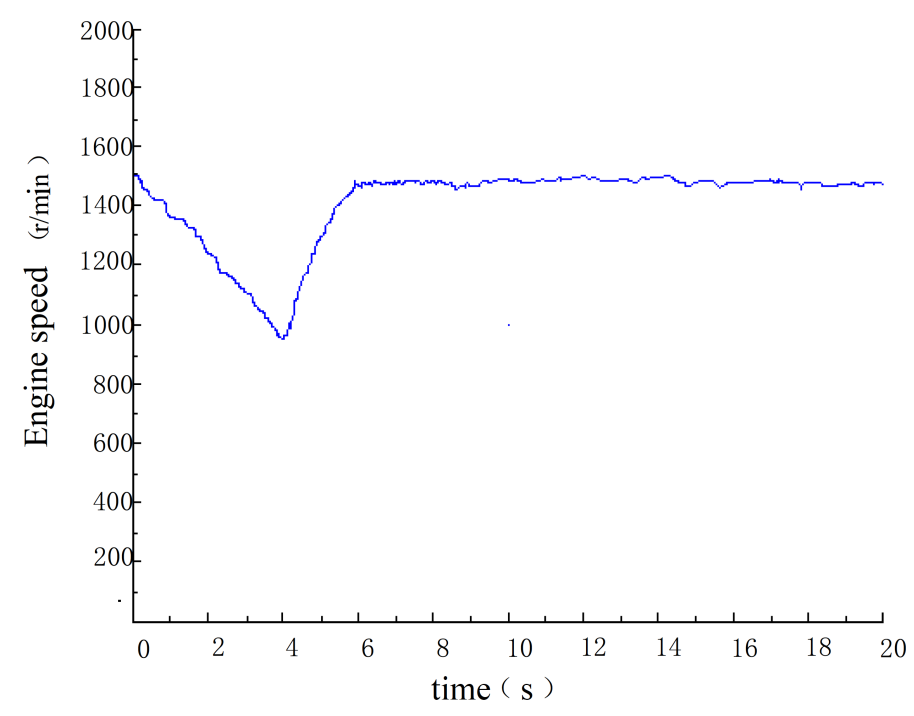

Fig. (6). The conversion curve of engine speed. 


\section{CONFLICT OF INTEREST}

The authors confirm that this article content has no conflict of interest.

\section{ACKNOWLEDGEMENTS}

The research was supported by the Importation and Development of High-Caliber Talents Project of Beijing Municipal Institutions under the Grant IDHT201304086

\section{REFERENCES}

[1] Y.T. Rao, J.J. Zou, J.H. Wang, "Field bus CAN Principles and Applications of Technology", Beijing University of Aeronautics and Astronautics Press, 2003, p. 6.

[2] K.M. Wu, "CAN Bus Principle and Application System Design", Beijing University of Aeronautics and Astronautics Press, 2002. 4ix ed.
[3] J.K. Han, Y. Jiang, "Application of CAN bus technology in hydraulic hybrid vehicles", Transactions of the Chinese Society for Agricultural Machinery, vol. 36, no. 9, pp. 152-153, 2005.

[4] B. Xie, E.R. Mao, "Development of CAN intelligent nodes of tractor electronic hydraulic hitch system", Transactions of the Chinese Society for Agricultural Machinery, vol. 37, no. 12, pp. 1-3, 2006.

[5] B. Xie, E.R. Mao, "Development of electronic hydraulic hitch controller based on CAN bus", Machine Tool and Hdraulics, vol. 34, no. 5, pp. 185-187, 2006

[6] S.Y. Bei, "Research and development of vehicle braking performance detection system", Instrumentation Technology, vol. 33, no. 6, pp. 16-20, 2004.

[7] S.F. Du, X.Z. Cao, J. Xu , "CAN Bus Measurement \& Control Technology and its Application", Electronic Industry Press, $2^{\text {nd }}$ ed., 2007.

[8] H.P. Liu, W.J. Wang and L. Deng, "TMS320LF240XA C Language Development and Application of DSP", Beijing University of Aeronautics and Astronautics Press, 2003, p. 9. 